\title{
Atypical presentation of Neuroleptic Malignant Syndrome associated with Lurasidone: a case report
}

\author{
Sumati Arikera ${ }^{1}$, Kartik Vangal ${ }^{2}$, Nayana Naik ${ }^{3}$ \\ ${ }^{1}$ Junior Resident, \\ ${ }^{2}$ Senior Resident, \\ ${ }^{3}$ Consultant, \\ Institute of Psychiatry and Human Behavior, Goa \\ Corresponding author: Sumati Arikera \\ Email: sumati.arikera@gmail.com
}

\begin{abstract}
Neuroleptic malignant syndrome (NMS) is a known life-threatening idiosyncratic reaction to antipsychotic drugs characterized by fever, altered mental status, muscle rigidity, and autonomic dysfunction. A vast literature is widely available on first generation antipsychotics causing NMS; however, it is also known that second generation antipsychotics are not spared from causing NMS. Drugs such as Olanzapine, Risperidone, Aripiprazole, Paliperidone causing NMS has been reported, although not much literature is currently available on NMS associated with Lurasidone. Hence to add to this literature, we present a case report of a patient who developed an atypical presentation of NMS associated with Lurasidone. Early recognition of the syndrome, immediate discontinuation of the offending agent, and prompt treatment with Bromocriptine produced a good recovery. The various features of the case are discussed in view of the potential lethality of the syndrome.
\end{abstract}

Keywords: Neuroleptic Malignant Syndrome, Lurasidone, Atypical antipsychotic

[Paper received $-30^{\text {th }}$ November 2020, Peer review completed $-10^{\text {th }}$ December 2020]

[Accepted - $18^{\text {th }}$ December 2020]

\section{INTRODUCTION}

NMS can be caused by a wide range of drugs, mainly Haloperidol, Chlorpromazine (reported to be having the highest risk), Clozapine, Quetiapine, Ziprasidone [1], Levodopa [2], Metoclopramide [3], Amoxapine and Tetrabenazine [4]. The first reported case of NMS appeared in 1956, shortly after the introduction of the antipsychotic drug chlorpromazine (Thorazine) [5].

Only a few cases of NMS associated with Lurasidone have been reported worldwide, and having been recently launched in India, it lacks the post-marketing surveillance data on its association with NMS in our country. Hence, we report this case report of NMS associated with Lurasidone which, to the best of our knowledge, is the first such case report in India.

The underlying pathophysiologic mechanisms of NMS are known to be complex and are still a topic of debate amongst experts. However, the most agreed mechanism is of marked and sudden reduction in central dopaminergic activity resulting from the D2 receptor blockade within the nigrostriatal, hypothalamic, mesolimbic/cortical pathways, which explains the rigidity, hyperthermia, and altered mental status respectively [6-7]. However, D2 receptor antagonism on its own does not explain all the symptoms and signs of NMS. Neither does it explain its occurrence with antipsychotic medications without known antidopaminergic activity. Hence this has led to a proposal by some that there is sympathoadrenal hyperactivity, which happens due to removal of tonic inhibition within the sympathetic nervous system [8]. These abnormalities in the sympathetic system are supported by the frequent presence of autonomic 
symptoms in NMS, and the changes in urine and plasma catecholamine levels in patients with NMS. One theory is that NMS shares pathophysiological similarities with malignant hyperthermia and a defect in calcium regulatory proteins within sympathetic neurons may be the key factor that brings about the onset of NMS [9]. Another theory also suggests the role of peripheral skeletal muscle system, the release of calcium has been shown to be increased from the sarcoplasmic reticulum of muscle cells with antipsychotic usage, possibly leading to increased muscle contractility and rigidity, breakdown of muscle and hyperthermia [6]. However, none of the theories stated above can explain why only a fraction of patients exposed to neuroleptics develop NMS.

The diagnosis of NMS is based on history and certain physical findings and laboratory investigations. Patients typically develop NMS within hours or days after exposure to a causative drug, with most exhibiting symptoms within 2 weeks and nearly all within 30 days [10]. NMS has classically been characterized by the presence of the triad of fever, muscle rigidity, and altered mental status, but its presentation can be quite heterogeneous and can include tachycardia, tachypnea, altered mental status, mutism, incontinence, diaphoresis, hyper salivation. However, all the features may not be present in every patient of NMS; a few of them could have only a few features giving a confusing picture of the situation.

Characteristic laboratory findings seen in NMS include elevated creatinine phosphokinase (CPK) due to rhabdomyolysis and leucocytosis, but these are neither specific for the syndrome nor present in all cases [11]. The cerebrospinal fluid (CSF) and imaging studies are usually normal, but an electroencephalogram (EEG) may show non-generalized slowing [10]. Lurasidone is an antagonist of dopamine D2 and D3 receptors, serotonin 5-HT2A and 5-HT7 receptors, and the alpha 2C adrenergic receptor, and a partial agonist of the serotonin 5-HT1A receptor. Although NMS occurs only rarely, it remains an unpredictable and potentially life- threatening neurological condition that clinicians must be able to recognize, since early identification and proper medical management are essential to ensure improved patient outcomes.

\section{CASE REPORT}

A 26-year-old male patient, native of Tamil Nadu was brought as Magistrate Order to our hospital in May 2020 as he had aggressive and destructive behaviour in the protective home that he was placed in. Upon admission the patient was started on Risperidone $2 \mathrm{mg}$ /day which was gradually increased to $6 \mathrm{mg} /$ day. Since the patient showed no improvement on Risperidone, it was tapered and stopped. Olanzapine was started which was gradually increased to $30 \mathrm{mg} /$ day. Patient developed persistent hypotension, hence Olanzapine was tapered and stopped. Subsequently the patient was started on Amisulpride $100 \mathrm{mg} /$ day to which he developed EPS at a dose of $150 \mathrm{mg} /$ day which was increased to $800 \mathrm{mg} /$ day and was continued for around 2 months. Due to partial response to Amisulpride, Lurasidone was added at the dose of $40 \mathrm{mg} /$ day, which was increased to $80 \mathrm{mg} /$ day after 4 days. The following day the patient had an episode of vomiting, urinary incontinence, neck stiffness, he appeared to be confused, in altered sensorium and was immediately shifted to Medicine casualty where meningitis was suspected. He was admitted under the Department of Medicine and was investigated for neurological causes. Lumbar puncture was done for cerebrospinal fluid analysis, which was within normal limits, Computerised Tomography of Brain was within normal limits, RT-PCR for SARSCoV- 2 was done which was negative. Injectable antibiotics were given, and antipsychotics were stopped. The patient was transferred back to Psychiatry three days later, for suspected catatonia. On examination he was found to be mute, he had mild rigidity, and urinary incontinence which aroused the suspicion of Neuroleptic Malignant Syndrome. Laboratory investigations showed normal leukocyte count (7,700) (normal range 4,000-11,000 WBCs per microliter), elevated Aspartate Aminotransaminase level (54U/L) (normal range 5-40U/L). The patient had marked elevation of Creatine Kinase (22526 U/L) (normal range 22-198 U/L), with elevated CK-MB fraction (28U/L) (normal range 5-25 U/L) and normal Cardiac Troponin I level $(<0.40 \mathrm{ng} / \mathrm{ml})$. ECG revealed no acute ischaemic changes. Hence the patient was referred and readmitted under the Department of Medicine and was started on Bromocriptine $2.5 \mathrm{mg}$ twice a day. He was admitted for 8 days during which supportive treatment was given and he was discharged on Bromocriptine $2.5 \mathrm{mg}$ once a day for seven days. Patient recovered well, creatine kinase level at the time of discharge was 555U/L. Currently the patient is stable and maintaining well 


\section{DISCUSSION}

NMS is not a dose-dependent phenomenon. Rather, it is an idiosyncratic reaction to antipsychotic drugs that may occur after a single dose or one taken in the same quantity for many years. It may also result from a dosage that is too high or increased too quickly. An idiosyncratic reaction, or type B reaction, is an unpredictable adverse reaction to a drug that is not the consequence of a known pharmacologic property or patient allergy. Instead, it is an abnormal response to normal drug dosages that occurs unexpectedly due to genetic factors [12-13]. Symptom onset from these reactions typically occurs between 4 and 14 days after the initiation of a pharmacologic therapy, with $90 \%$ of patients developing symptoms within 10 days [13].

Our patient was tried on Risperidone $6 \mathrm{mg} /$ day to which he did not respond, Olanzapine $30 \mathrm{mg} /$ day to which he developed persistent hypotension and hence was stopped. He was started on Amisulpride at the dose of $100 \mathrm{mg} /$ day to which he developed EPS at a dose of $150 \mathrm{mg} /$ day, which was gradually increased to $800 \mathrm{mg} /$ day for a duration of 2 months. Due to partial response to the same, Lurasidone was added at 40 $\mathrm{mg}$. After 4 days of its initiation the dose was increased to $80 \mathrm{mg} /$ day. The following day the patient had an episode of vomiting, appeared confused and delirious, had neck stiffness, and was transferred to the department of medicine. The patient then developed signs of catatonia and was referred to Psychiatry. Patient had developed mutism and rigidity due to which we suspected NMS and hence got the creatinine phosphokinase level which was found to be very high $(22526 \mathrm{U} / \mathrm{L})$ and hence was readmitted in the medicine ward after the opinion of the neurologist was taken. Patient was treated as a case of NMS, after which he improved and was transferred to Psychiatry.

According to Naranjo's algorithm, a questionnaire designed by Naranjo et al, to determine the likelihood of whether an adverse drug reaction is due to the drug rather than the result of other factors, it fulfilled the criteria of 'probable' NMS associated with Lurasidone [14]. The possible risk factors for this patient to develop NMS could be due to the initiation or increase in the dose of Lurasidone with male gender and young age being the other risk factors [15-16]. The above patient recovered significantly after starting Bromocriptine (Dopamine agonist) and other supportive treatment. At the time of discharge from the medicine ward he was fully conscious, oriented, and had no rigidity. Patient has no psychotic symptoms. If patient develops any psychotic symptoms the plan is to start him on Clozapine, as the available literature states that recurrences of NMS do occur, especially when the patient is restarted with a neuroleptic with high potency or too quickly after the initial episode [6, 17-18]. We consider starting Clozapine as it is generally felt to be prudent to use a different neuroleptic agent than the one that was originally associated with the development of the syndrome [6, 19-20]. NMS is an idiosyncratic reaction and the percentage of NMS due to Lurasidone during the premarketing evaluation was found to be $0.1 \%$.[21]. Hence it is imperative that when psychiatrists prescribe Lurasidone, they should be vigilant about the development of NMS even on lower doses, so that timely treatment can be given thus preventing mortality and reducing morbidity due to NMS.

\section{Declaration of patient consent}

The authors certify that they have obtained all appropriate patient consent forms. The patient has consented for; his clinical information will be used to report in a journal. The patient also understands that his name and other initials will not be disclosed, and due efforts will be made to conceal his identity.

\section{REFERENCES}

1. Khaldi S, Korneich C, Choubani Z, Gourevitch R. Neuroleptic malignant syndrome and atypical antipsychotics:a brief review. L'Encephale 2008;34(6):618-24.

2. Keyser DL, Rosnitzky RL. Neuroleptic malignant syndrome in Parkinson's disease after withdrawal or alteration of dopaminergic therapy. Arch Intern Med 2008;151(4):794-6.

3. Friedman LA, Weinrauch LA, D'Elia JA. Metoclopramide induced neuroleptic malignant syndrome. Arch Intern Med 1987;147(8):1495-7.

4. Buckley PF, Hutchinson M. Neuroleptic malignant syndrome. J Neurol Neurosurg Psychiatry 2002;58(3):271-3.

5. Ayd F. Fatal hyperpyrexia during chlorpromazine therapy. J Clin Exp Psychopathol 1956;17(2):189-92. 
6. $\quad$ Adnet P, Lestavel P, Krivosic-Horber R. Neuroleptic malignant syndrome. Br J Anaesth 2000;85(5):12935

7. Strawn JR, Keck PE, Caroff SN. Neuroleptic malignant syndrome. Am J Psychiatry 2007;164(5):870-6.

8. Gurrera RJ. Sympathoadrenal hyperactivity and the aetiology of neuroleptic malignant syndrome. Am J Psychiatry 1999;156(2):169-80.

9. Gurrera RJ. Is neuroleptic malignant syndrome a neurogenic form of malignant hyperthermia? Clin Neuropharmacol. 2002;25(4):183-93

10. Caroff SN, Mann SC. Neuroleptic malignant syndrome. Psychopharmacol Bull 1988;24(1):25-9.

11. Caroff SN, Mann SC. Neuroleptic malignant syndrome. Med Clin North Am 1993;77(2):185-202.

12. Gurrera RJ. A systematic review of sex and age factors in neuroleptic malignant syndrome diagnosis frequency. Acta Psychiatr Scand 2017;135(5):398-408.

13. Pileggi DJ, Cook AM. Neuroleptic malignant syndrome. Ann Pharmacother 2016;50(11):973-81.

14. Naranjo CA, Busto U, Sellers EM, Sandor P, Ruiz I, Roberts EA, Janecek E, Domecq C, Greenblatt DJ. A method for estimating the probability of adverse drug reactions. Clin Pharmacol Ther 1981;30(2):23945.

15. Berardi D, Amore M, Keck PE, Troia M, Dell'Atti M. Clinical and pharmacologic risk factors for neuroleptic malignant syndrome: a case control study. Biol Psychiatry. 1998;44(8):748-54

16. Keck PE, Pope HG, Cohen BM, McElroy SL, Nierenberg AA. Risk factors for neuroleptic malignant syndrome - a case control study. Arch Gen Psychiatry 1989;46(6);914-8.

17. Pope HG, Aizley HG, Keck PE, McElroy SL. Neuroleptic malignant syndrome - long term follow up of 20 cases. J Clin Psychaitry 1991;52(5):208-12.

18. Susman VL, Addonizio G. Recurrences of neuroleptic malignant syndrome. J Nerv Ment Dis 1988;176(2):234-41.

19. Bhanushali MJ, Tuite PJ. The evaluation and management of patients with neuroleptic malignant syndrome. Neurologic Clin 2004;22(2):389-411

20. Shalev A, Hermesh H, Munitz, ,Munitz H. Mortality from neuroleptic malignant syndrome. J Clin Psychiatry. 1989;50(5):18-25.

21. Wellrx.com. Latuda: Clinical Pharmacology Drug Monograph; 2021.

$$
\begin{gathered}
\text { Acknowledgements - Nil } \\
\text { Conflict of Interest - Nil } \\
\text { Funding - Nil }
\end{gathered}
$$

